\title{
O trabalhador sem formação em enfermagem atuando em centro de material e esterilização: desafio para o enfermeiro
}

\author{
THE WORKER WITH NO FORMATION IN NURSING IN A CENTER OF MATERIAL \\ AND STERILIZATION: A CHALLENGE TO THE NURSE
}

\author{
EL TRABAJADOR SIN FORMACIÓN EN ENFERMERÍA ACTUANDO EN CENTRO \\ DE MATERIAL Y ESTERILIZACIÓN: DESAFIO PARA EL ENFERMERO
}

\section{Anaclara F. Veiga Tipple ${ }^{1}$, Thays Ribeiro de Souza², Ana Lúcia Queiroz Bezerra ${ }^{3}$, Denize Boutellet Munari ${ }^{4}$}

\section{RESUMO}

$\mathrm{O}$ estudo teve com objetivo caracterizar o processo de inserção nos Centros de Material e Esterilização - CME, de trabalhadores que não possuem formação específica na área de enfermagem. Fizeram parte do estudo 75 trabalhadores de dois hospitais públicos. Após observação dos aspectos éticos legais, os dados foram obtidos por meio de questionário previamente validado e testado. Foram encontrados 15 trabalhadores sem formação específica, provenientes de serviços diversos intra e extra hospitalar, os quais não foram submetidos a processos de seleção ou treinamentos, tendo aprendido a rotina do serviço com colegas de trabalho. Tais aspectos são preocupantes, considerando-se o risco ocupacional do trabalhador, o comprometimento da qualidade do reprocessamento de artigos hospitalares e o cumprimento da legislação do exercício profissional.

\section{DESCRITORES}

Recursos humanos de enfermagem. Prática profissional.

Educação continuada.

\begin{abstract}
The object of this study was to characterize the process of insertion in the Centros de Material e Esterilização - Center of Material and Sterilization (CME) of workers who have no specific formation in the area of Nursing. Seventy-five workers of two public hospitals took part in the study. After the observation of the legal ethical aspects, data was obtained through a questionnaire previously validated and tested. The study found 15 workers without specific formation, who came from different intra- and extra-hospital services. They had not been submitted to selection processes nor to training, and had learned the work routine with co-workers. This is worrisome, considering the worker's occupational risk, the risks this situation represents for the quality of the reprocessing of hospital items and the conformity to the legislation that governs professional practice.
\end{abstract}

\section{KEY WORDS}

Nursing Staff.

Professional practice.

Continuing education.

\section{RESUMEN}

Para este estudio se tuvo como objetivo caracterizar el proceso de inserción en los Centros de Material y Esterilización - CME, de trabajadores que no poseen formación específica en el área de enfermería. Hicieron parte del estudio 75 trabajadores de dos hospitales públicos. Después de la observación de los aspectos éticos legales, los datos fueron obtenidos por medio de un cuestionario previamente validado e testado. Fueron encontrados 15 trabajadores sin formación específica, provenientes de servicios diversos intra y extrahospitalarios, los cuales no fueron sometidos a procesos de selección o entrenamientos, habiendo aprendido la rutina del servicio con colegas del trabajo. Tales aspectos son preocupantes, considerándose el riesgo ocupacional del trabajador, el compromiso de la calidad del reprocesamiento de artículos hospitalarios y el cumplimiento de la legislación del ejercicio profesional.

\section{DESCRIPTORES}

Personal de enfermería. Práctica profesional. Educación continua.
1 Professor Adjunto da Faculdade de Enfermagem da Universidade Federal de Goiás - UFG. anaclara@fen.ufg.br

2 Enfermeira graduada pela Faculdade de Enfermagem da UFG.

3 Professor Adjunto da Faculdade de Enfermagem da Universidade Federal de Goiás - UFG.

4 Professora Titular da Faculdade de Enfermagem da UFG. Pesquisadora do CNPq. 
Anaclara F. Veiga Tipple Thays Ribeiro de Souza Ana Lúcia Queiroz Bezerra Denize Boutellet Munari

\section{INTRODUÇÃO}

O Centro de Material e Esterilização - CME é definido como o conjunto de elementos destinados a recepção e expurgo, preparo e esterilização, guarda e distribuição do material para as unidades de estabelecimento de saúde; sendo que a existência desse serviço está ligada a própria história da evolução da cirurgia, quando os procedimentos cirúrgicos eram realizados somente em local inapropriado, sem se considerar cuidados básicos de higiene, com os instrumentais do tipo agulhas, bisturis e demais artigos ${ }^{(1)}$.

Com o desenvolvimento das técnicas cirúrgicas, foi crescente a necessidade de se aprimorar materiais e equipamentos utilizados, exigindo assim cuidados especiais no trato com os mesmos. Cientistas como Pasteur e Robert Koch proporcionaram à ciência, grande conhecimento na área de microbiologia; enfatizou-se a desinfecção de instrumentais cirúrgicos, lavagem das mãos dos cirurgiões, com o intuito de prevenir o desenvolvimento de bactérias patogênicas. Nesse contexto, já se iniciavam as normas técnicas, rotinas e procedimentos a serem aplicados aos artigos hospitalares ao ambiente dos trabalhadores, que foram gradativamente sendo melhoradas e aperfeiçoadas, desta forma, originando as atuais rotinas a serem implementadas em um CME.

Para compreender a contextualização do CME no processo de controle de infecção, basta atentar-se ao fato da utilização dos artigos odontomédico-hospitalares sem o devido comprometimento dos serviços prestados ao cliente. É necessário que tais artigos tenham previamente passado por um fluxograma unidirecional que se resume em limpeza, secagem, preparo e esterilização e estocagem. Qualquer falha ocorrida durante o processamento implica em possíveis complicações como, por exemplo, infecção trans ou pósoperatória ${ }^{(2)}$.

Segundo o Manual de Prevenção e Controle de Infecção Hospitalar da Bahia ${ }^{(3)}$, o processamento de artigos em CME ocupa um lugar importante no hospital, estando relacionado com a qualidade do produto final. Esse setor interfere significativamente no controle das infecções hospitalares, visto que o paciente hospitalizado dificilmente não experimenta um procedimento invasivo, o qual, por mais simples que seja, pode provocar o rompimento de barreiras naturais ou penetrar em cavidades estéreis. Se o instrumental a ser utilizado tiver sido reprocessado inadequadamente, o mesmo se tornará automaticamen- te, uma fonte de contaminação e transmissão de microrganismos.

Para a garantia da qualidade dos serviços prestados em CME é necessário que os recursos humanos tenham perfil adequado para tal fim, bem como, a devida capacitação teórico-prática ${ }^{(4-5)}$.

O treinamento é um processo educacional, aplicado de maneira sistemática e organizado, por meio do qual, as pessoas adquirem novos conhecimentos, atitudes e habilidades em função dos objetivos institucionais ${ }^{(6)}$. No CME o treinamento torna-se uma ferramenta indispensável para a capacitação dos funcionários no trabalho, pois proporciona-lhes segurança, pela aquisição de hábitos de reflexão e ação, ampliando o nível de qualificação do seu desempenho.

Ao contrário do que se observa na realidade, a seleção de pessoal para o CME, deve seguir critérios rigorosos. Os trabalhos desempenhados nesse setor exigem técnica, zelo e muita responsabilidade. A qualidade do pessoal tem então, notável influência no funcionamento da unidade e na prevenção e no controle das infecções hospitalares.

O trabalhador do CME deve ser atento e organizado, gostar do que faz, e saber executar corretamente o que faz. Assim, para que tal exigência seja cumprida, o trabalhador deve ter cursado no mínimo o ensino fundamental, além de ter formação específica na área de enfermagem ${ }^{(4-5)}$.

De acordo com as recomendações do Ministério da Saúde ${ }^{(7)}$, as pessoas selecionadas para trabalhar nessa unidade devem receber treinamento condizente com a função, inspirar confiança e credibilidade, saber planejar, organizar, ser atento, ter postura profissional e manter a cadeia asséptica.

Nesse sentido, a existência de uma política de educação continuada como estratégia de desenvolvimento do pessoal de enfermagem do CME tem demonstrado importância na melhoria da qualidade desses serviços nas instituições de saúde, visto que a realidade exige do indivíduo conhecimentos atualizados, que só a escola não está apta a fornecer ${ }^{(8)}$.

Portanto, a complexidade dos processos de esterilização e alto custo na aquisição de instrumentais cirúrgicos cada vez mais sofisticados, exigem investimentos na qualificação do profissional. Ter pessoas qualificadas significa diminuição dos índices de infecção hospitalar, do tempo de internação, e conseqüentemente, redução de gastos $^{(1)}$. 


\section{OBJETIVO}

Caracterizar o processo de inserção nos Centros de Material e Esterilização - CME, de trabalhadores que não possuem formação específica na área de enfermagem.

\section{MÉTODO}

Estudo descritivo, desenvolvido em duas instituições hospitalares públicas do município de Goiânia, com mais de 100 leitos, as quais possuíam trabalhadores em CME sem formação específica em enfermagem. No ano de 2003, na cidade de Goiânia existia 12 hospitais com número de leitos superior a 100. Sendo que, dois destes não consentiram em participar do estudo e, em oito não foram encontrados trabalhadores sem formação na área de enfermagem.

A população constituiu-se de 75 trabalhadores dos CME das duas instituições, que se dispuseram a participar da pesquisa após serem informados dos objetivos da mesma. A Os dados foram obtidos no mês de janeiro de 2003, mediante a aplicação de um questionário previamente submetido a teste piloto.

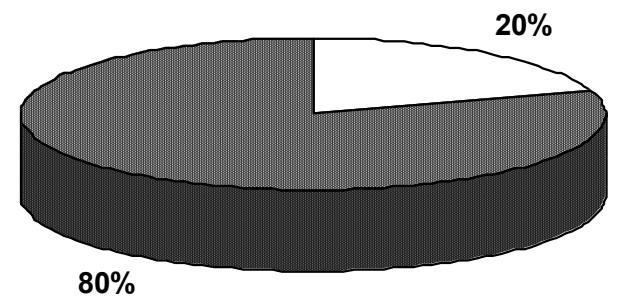

Os trabalhadores foram abordados durante o turno de trabalho, conforme a disponibilidade de cada um, sendo que ninguém se recusou a responder ao questionário. Na ocasião da coleta de dados foi apresentado e assinado o Termo de Consentimento Livre e Esclarecido. O projeto foi aprovado pelo Comitê de Ética em Pesquisa Médica Humana e Animal do Hospital das Clínicas da Universidade Federal de Goiás, conforme preconizada pela Portaria 196/96 do Conselho Nacional de Saúde.

Os dados foram submetidos à análise estatística descritiva e para sua apresentação foi considerada a pertinência da abordagem da temática, agrupando-os em: processo de inserção no trabalho e participação em programas de educação continuada.

\section{RESULTADOS E DISCUSSÃO}

Os dados que objetivaram caracterizar a população nos permitiu verificar que dos 75 trabalhadores de CME contatados, 15 (20,0\%) não possuíam formação específica em enfermagem e representam a amostra do estudo; 60 trabalhadores $(80,0 \%)$ tinham formação específica em enfermagem, conforme mostra a Figura 1.

$\square$ Sem form. espec. em Enf.

圆 Com form. espec. em Enf.
O trabalhador sem formação em enfermagem atuando em centro de material e esterilização: desafio para o enfermeiro

Figura 1- Distribuição dos trabalhadores de CME de duas instituições públicas, segundo a formação na área de enfermagem. (Goiânia, 2003)

A manipulação de material contaminado é certamente um risco constante, ainda que observadas todas as técnicas de manuseio e normas de segurança ${ }^{(9)}$. Assim, esse risco estaria multiplicado quando o trabalhador, sem formação técnicocientífica consistente, sem bases de assepsia e antisepsia ou noções de microbiologia e biossegurança, reprocessa artigos odonto-médico-hospitalares. Entretanto, muitas vezes esta tarefa é delegada a pessoas não capacitadas e não treinadas para tal fim ${ }^{(10)}$

Admitir mão-de-obra sem formação específica em enfermagem, para executar as atividades pertinentes ao CME, implica em prejuízos ao cliente que procura pelos serviços de saúde, ao enfermeiro responsável pelo setor, e ao próprio trabalhador em questão, visto que falhas humanas nos processos de limpeza, preparo, desinfecção ou esterilização e acondicionamentos de artigos, podem resultar em riscos aos clientes, como os de infecção hospitalar. Por outro lado os riscos ocupacionais se elevam pelo manuseio incorreto de material contaminado. $\mathrm{O}$ enfermeiro supervisor é quem responde legalmente por qualquer dano causado ao trabalhador, resultante da falta de aptidão ou capacitação técnica. Age com imprudência ao aceitar profissionais desqualificados ${ }^{(9)}$.

O trabalhador de CME com formação específica e devidamente treinado para atuar neste setor, certamente refletiria melhor diante de ações, como

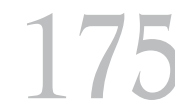

Rev Esc Enferm USP 2005; 39(2):173-80 
Anaclara F. Veiga Tipple Thays Ribeiro de Souza Ana Lúcia Queiroz Bezerra Denize Boutellet Munari a não realização criteriosa da limpeza dos artigos que interfere diretamente na esterilização, considerada a mais importante medida de proteção antiinfecciosa e que se relaciona diretamente com o controle das infecções hospitalares.

Desta forma, o despreparo do trabalhador do CME implica em imperícia, negligência e/ou imprudência no tocante às atividades pertinentes ao serviço, colocando em risco a qualidade do atendimento prestado ao cliente.

Tais achados são motivos de preocupação, pois admitir mão-de-obra sem formação específica em enfermagem, aos quadros da enfermagem do $\mathrm{CME}$, contraria não apenas nossos esforços em manter a enfermagem exercida por profissionais legalmente habilitados, mas também burla de maneira sutil a lei que restringe a atividade dos profissionais sem formação específica ${ }^{(9)}$. De nada adiantará o CME adquirir produtos e equipamentos que prometam milagres, se esses não forem utilizados por uma equipe treinada a respeito da ciência do reprocessamento de $\operatorname{artigos}^{(10)}$.

O CME deve estar sob a responsabilidade de enfermeiros capacitados, que realizem a orientação e supervisão de todas as etapas do reprocessamento de $\operatorname{artigos}^{(11)}$. $\mathrm{O}$ estudo identificou a presença de enfermeiros ocupando cargos de gerência em todos os CME, o que é esperado pela instituição, para garantir a qualidade e produtividade do serviço.

A função do enfermeiro do CME tem início na fase de planejamento da unidade. A ele cabe a escolha dos recursos materiais e humanos condizentes com as atividades do setor. Este tem total responsabilidade pela seleção e treinamento de pessoal; tanto a qualificação quanto o recrutamento dos recursos humanos devem ser dimensionados criteriosamente, levando em conta o trabalho e o funcionamento do $\mathrm{CME}^{(2)}$.

Assim, entende-se que o gestor de enfermagem dos CME que compuseram o estudo, mesmo sendo o responsável pelo recrutamento e seleção de pessoal, e teoricamente com autonomia para esta atividade, ainda contempla no seu quadro de pessoal, trabalhadores sem formação específica para desenvolver atividade de enfermagem no setor.

Ao contrário do que se observa na realidade pesquisada, a seleção de pessoal para o CME deve obedecer a critérios rigorosos, pois os trabalhos realizados nesse setor exigem técnica, zelo e muita responsabilidade, requerendo trabalhadores atentos, organizados, e que sabem executar corretamente as atividades que lhe cabem.

\section{Processo de Inserção no Trabalho / Treinamento}

O programa de treinamento oferecido pela instituição em uma área específica é um facilitador para a inserção do indivíduo e para o desenvolvimento da prática profissional no ambiente de trabalho, para que ele exercite a reflexão sobre a importância desse trabalho no seu dia a dia.

Quanto à ocupação anterior dos trabalhadores do CME sem formação específica na área de enfermagem, verificaram-se sete trabalhadores $(46,7 \%)$ provenientes dos serviços de higienização e limpeza hospitalar, quatro $(26,6 \%)$ de serviços de portaria, três $(20,0 \%)$ de lavanderias hospitalares e um $(6,7 \%)$ que, antes de ser admitido, exercia apenas serviços domésticos, conforme mostra a Figura 2.

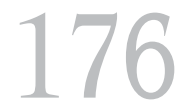

Rev Esc Enferm USP 2005; 39(2):173-80

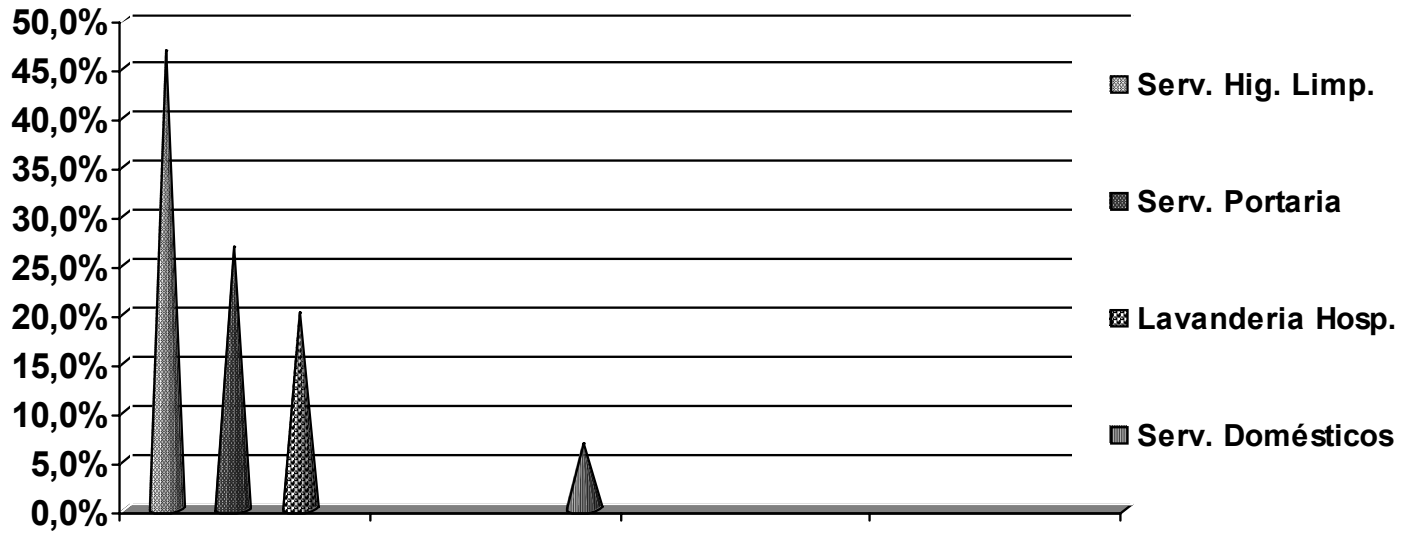

Figura 2 - Distribuição dos trabalhadores do CME quanto à área de atuação antes da admissão no CME. (Goiânia, 2003) 
Quando indagados sobre os critérios de seleção aos quais foram submetidos para admissão no CME, todos $(100,0 \%)$ referiram ter sido por opção da chefia de enfermagem, sendo que $14(93,3 \%)$ foram transferidos de outros setores e um $(6,7 \%)$ não tinha vínculo com a instituição. Os mesmos informaram que não foram entrevistados ou avaliados previamente, pela chefia do CME e que nunca participaram de programas de treinamento sobre CME; tendo aprendido as rotinas do serviço com os colegas, mais experientes.

Essa é uma prática que pode garantir procedimentos uniformes, padronizados, mas pode incorrer na repetição de vícios, comuns nessas unidades. Portanto, o treinamento deve ser realizado pelo enfermeiro, além de constituí-se em um momento de interação com o funcionário recém-admitido, poderá minimizar possíveis falhas que poderão ocorrer nos processos de trabalho.

Para o Ministério da Saúde ${ }^{(12)}$, o descaso com a política de recursos humanos explica boa parte das dissonâncias que se observam na área da saúde em muitos países. As mudanças na composição da força de trabalho, nos processos de sua formação e regulação profissional, nas regras de contratação são incógnitas que representam desafios para a formulação, acompanhamento e avaliação das políticas de recursos humanos nos cenários do país. A percepção dessas mudanças, ou pelo menos de suas tendências constitui atributo indispensável para os enfermeiros gestores dessas políticas. Desse modo, torna-se indispensável desenvol-ver metodologias que permitam aferir essas tendências e estimar as mudanças que devem ser aí introduzidas ${ }^{(13)}$

Além disso, convém enfatizar a importância de se tentar cada vez mais qualificar o trabalho do CME e seus membros, abandonando o voluntarismo e o conseqüente amadorismo. Porque o mercado de trabalho está desejoso de profissionais com qualificados, que saibam agir, tomar decisões e usar da criatividade; é necessário priorizar tais qualidades no momento do recrutamento de profissionais.

Agregar mão de obra desqualificada a um serviço de alta complexidade, que envolve diversos segmentos da estrutura hospitalar pode implicar em descrédito nas ações de enfermagem em geral, além de danos ao cliente que busca pela assistência a saúde.

No início da década de 90, a assistência de enfermagem no Brasil era feita em grande parte, por um contingente de pessoas sem preparo formal para o trabalho. Tal situação, determinada social e historicamente trazia distorções na prática de enfermagem e na produção dos serviços de saúde. Em função disso, esses serviços se mostraram ineficazes, dispendiosos, pouco produtivos e até mesmo iatrogênicos ${ }^{(14)}$. É lamentável constatar-se que neste novo milênio, essa situação ainda permanece em setores de relevada importância no contexto hospitalar.

As instituições de saúde são empresas que prestam serviços aos clientes, e devem se conscientizar que as mudanças são importantes, pois estão sendo avaliadas não só pelo custo, mas pela qualidade do produto ${ }^{(15-17)}$. Logo, maior rigor no tocante à seleção de mão de obra resultará em prestação de assistência com qualidade.

A enfermagem a exemplo dos hospitais, também deve começar a estudar e adotar o Gerenciamento de Qualidade com vistas a alcançar não só um padrão aceitável de assistência, mas também a atender às expectativas dos trabalhadores $\mathrm{e}$ dos clientes ${ }^{(15)}$.

Os recursos humanos são o maior patrimônio da qualidade, por isso deve-se selecioná-los e sua capacitação é um pré-requisito indispensável a qualquer instituição ${ }^{(18-19)}$.

A prática do sistema de saúde público e privado absorvia funcionários sem nenhum critério de avaliação. O desconhecimento do número total desses trabalhadores em todas as áreas demonstrava a incapacidade de se conhecer as conseqüências e impacto de seu recrutamento na organização do processo e na qualidade do produto do trabalho em saúde. Estimava-se que somente no estado de São Paulo, no que se refere à enfermagem, existiam cerca de 80 mil trabalhadores inseridos no mercado de trabalho sem qualificação formal ${ }^{(20)}$.

O Conselho Federal de Enfermagem ${ }^{(21)}$, no artigo $1^{\circ}$ da Lei $8.967 / 94$, dispõe como as únicas atividades de enfermagem passíveis de serem realizadas pelo pessoal sem formação específica em enfermagem, aquelas consideradas como elementares, que compreendem ações de fácil execução e entendimento, baseadas em saberes simples, sem requererem conhecimento científico, adquirido por meio de treinamento e/ou prática; não colocam em risco a comunidade o meio ambiente e a saúde do executante, mas contribuem para que a assistência de enfermagem seja mais eficiente.
O trabalhador sem formação em enfermagem atuando em centro de material e esterilização: desafio para o enfermeiro 
Anaclara F. Veiga Tipple Thays Ribeiro de Souza Ana Lúcia Queiroz Bezerra Denize Boutellet Munari

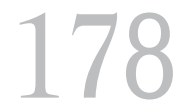

Rev Esc Enferm USP 2005; 39(2):173-80.
Os achados deste estudo contrariam as resoluções do COFEN, pois o CME envolve contato com artigos contaminados por material biológico, sendo que as atividades nele desenvolvidas representam risco para seus executores, não podendo ser consideradas como elementares, tampouco, não podem ser executadas por trabalhadores sem formação específica em enfermagem. Ademais, garantir o reprocessamento de artigos com qualidade é considerado como uma das principais medidas de proteção anti-infecciosa $^{(1)}$. Torna-se importante considerar a qualificação dos recursos humanos e sua relação com o controle de infecção hospitalar.

Nas potências mundiais, o Controle de Infecção Hospitalar é um pré-requisito que deve conter no currículo dos profissionais da saúde, portanto, estes chegam ao mercado de trabalho conscientes e preparados para o problema das infecções ${ }^{(22)}$.

A prevenção e controle de infecção devem nortear a política de formação dos profissionais da área da saúde. Não é aceitável nos dias atuais, dentro dos padrões éticos estabelecidos, dos paradigmas da qualidade da assistência e da qualidade de vida, qualquer profissional da saúde receber sua credencial profissional, seu diploma, sem ter uma base em prevenção e controle de infecção, sem ter um preparo técnico específico $^{(23)}$.

Um dos principais desafios enfrentados pelos que atuam no controle de infecção é estimular os profissionais de saúde a seguirem as recomendações preconizadas pelas Comissões de Controle de Infecção Hospitalar. Medidas aparentemente simples como a higienização das mãos, não são adotadas por um grande número de profissionais ${ }^{(24)}$

Uma forma de colocar à disposição do trabalhador os conhecimentos necessários para o desempenho do seu trabalho no CME (noções de assepsia, anti-sepsia, microbiologia, biossegu-rança e medidas de prevenção e controle de infecção hospitalar) seria subsidiar a participação deste no Projeto de Profissionalização dos Trabalhadores na área da Enfermagem - PROFAE ${ }^{(25)}$.

A criação do PROFAE contribuirá para qualificar esses trabalhadores que exercem sua profissão de forma irregular, diminuir os riscos à população atendida, melhorando a qualidade da atenção à saúde brasileira ${ }^{(25)}$.

\section{Participação em Programas de Educação Continuada}

A educação continuada é um processo permanente de práticas educativas planejadas no sentido de promover oportunidades de desenvolvimento pessoal e profissional dos indivíduos ${ }^{(26)}$.

Todos os trabalhadores pesquisados (100,0\%) referiram não terem participado de processo de educação continuada desde quando foram inseridos no CME. Quando indagados sobre sugestões para a melhoria do funcionamento do $\mathrm{CME}$, destacaram as palestras, programas de treinamentos, cursos, visando o enriquecer do conhecimento e reforço das habilidades.

Quanto à importância da participação do trabalhador em programas de educação continuada, devido às peculiaridades das atividades no CME, há dificuldades em se manter um grupo estável e em sincronia ${ }^{(2)}$. Para reverter ou prevenir essa situação, devem ser desenvolvidos programas contínuos de treinamento e aperfeiçoamento dos trabalhadores.

Sobre a educação continuada, a enfermagem deve proporcionar ao funcionário a oportunidade de atualizar seus conhecimentos; o treinamento deve ocorrer tanto de modo formal, por meio de cursos e palestras, como informal, pela orientação e discussões do dia a dia. Os funcionários recém admitidos ou transferidos devem receber treinamento condizente para que saibam o que são, e como fazer as atividades do setor. As pessoas nas instituições são consideradas como o mais importante patrimônio, por isso lhes deve ser oportunizado o crescimento profissional $^{(15)}$.

A rápida mudança no sistema de prestação de cuidados à saúde, a diversidade e complexidade crescente da prática exigem a instrumentalização da equipe, para que sejam capazes de pensar criticamente a fim de processarem dados e tomarem decisões oportunas em relação à assistência ao cliente ${ }^{(27)}$. Além disso, o ensino tem como principal função a busca pela competência profissional ${ }^{(28)}$.

Todavia, deve-se não só incentivar o aprendizado da equipe, mas também incentivar a prática daquilo que foi aprendido.

A chance de enriquecer os conhecimentos deve ser oferecida, porém, os interessados devem tomar iniciativa e estarem sempre atentos às possibilidades de atualização ${ }^{(29)}$. 
Por fim constata-se que os custos iniciais para implantação de um programa de educação continuada, envolvem principalmente o salário de um enfermeiro de Infecção Hospitalar. Além disso, outros gastos estão relacionados com treinamento e vigilância. Por outro lado, se a equipe está treinada, haverá retorno tanto no aprimoramento desta quanto na qualidade do atendimento ao paciente ${ }^{(30)}$.

O custo será compensado pela maior eficiência do funcionário no trabalho e pela produtividade, decorrentes de seu aprendizado ${ }^{(31)}$.

\section{CONCLUSÃO}

Este estudo realizado entre os trabalhadores de CME sem formação específica em enfermagem, de dois hospitais públicos da cidade de Goiânia concluiu que:

- o número total de trabalhadores sem formação específica em enfermagem identificados no estudo foi de 15;

- sete trabalhadores (46,7\%) atuavam nos serviços de higienização e limpeza hospitalar antes de serem inseridos no CME, quatro $(26,6 \%)$ em serviços de portaria, três $(20,0 \%)$ em lavanderias hospitalares e um $(6,7 \%)$ em serviços domésticos;
- todos os trabalhadores $(100,0 \%)$ referiram não terem sido submetidos a qualquer critério avaliativo quando admitidos no setor em questão;

- todos os trabalhadores $(100,0 \%)$ referiram nunca terem participado de programas de treinamento ou de educação continuada, tendo aprendido a rotina do serviço com os colegas de trabalho mais experientes.

Tais achados comprometem a qualidade da assistência prestada ao cliente e evidenciam que há trabalhadores sem formação específica em enfermagem, os quais nunca participaram de programas de educação continuada, reprocessando artigos odonto-médico-hospitalares.

Além disso, é fundamental que a chefia de enfermagem atue em conformidade com as recomendações do COFEN com relação á equipe de CME, não consentindo com o recrutamento de mão de obra desqualificada para atuar neste setor.

Considerar essas dimensões significa pensar nas exigências econômicas do processo de organização do trabalho que desvaloriza o saber técnico especializado, as exigências do mercado e da profissão.
O trabalhador sem formação em enfermagem atuando em centro de material e esterilização: desafio para o enfermeiro

\section{REFERÊNCIAS}

(1) Pinter MG, Gabrieloni MC. Central de material e esterilização. In: Fernandes AT, Fernandes MO, Ribeiro N, editores. Infecção hospitalar e suas interfaces na área da saúde. São Paulo: Atheneu; 2000. p. 1041 .

(2) Sales SMM. Central de material esterilizado. In: Oliveira AC, Albuquerque CP; Rocha LC M. Infecções hospitalares: abordagem, prevenção e controle. Rio de Janeiro: Medsi; 1998. p. 65-74.

(3) Secretaria de Estado da Saúde da Bahia. Coordenação de Desenvolvimento de Recursos Humanos. Departamento de Assistência à Saúde. Prevenção e controle de infeção hospitalar na Bahia. Orientações básicas. Salvador; 1998.

(4) Sociedade Brasileira de Enfermeiros de Centro Cirúrgico, Recuperação Anestésica e Centro de Material e Esterilização. Manual de práticas recomendadas da SOBECC. São Paulo; 2001.

(5) Sociedade Brasileira de Enfermeiros de Centro Cirúrgico, Recuperação Anestésica e Centro de Material e Esterilização. Manual de práticas recomendadas da SOBECC. $2^{\mathrm{a}}$ ed. São Paulo; 2003.

(6) Chiavenato I. Recursos humanos. $5^{\text {a }}$ ed. São Paulo: Atlas; 1999.
(7) Ministério da Saúde. Coordenação de Controle de Infecção Hospitalar. Processamento de artigos e superfícies em estabelecimentos de saúde. $2^{\text {a }}$ ed. Brasília; 1994.

(8) Bezerra ALQ. O treinamento do enfermeiro recémadmitido: visão do treinando e do treinador. [dissertação] São Paulo (SP): Escola de Enfermagem da USP; 1995.

(9) Leifert RMC. Auxiliares de esterilização. [cartas]. Rev SOBECC 1997; 2(4):26.

(10) Graziano KU. A importância dos procedimentos de limpeza nos processos de desinfecção e esterilização de artigos. Rev SOBECC 2002; 7(3):19-23.

(11) Molina E. O Centro de Material. In: Rodrigues EAC, Mendonça JS, Amarante JMB, Alves Filho MB, Grinbaum RS, Richtmann R. Infecção hospitalar: prevenção e controle. São Paulo: Sarvier; 1997. p. 491.

(12) Ministério da Saúde. Secretaria de Políticas de Saúde. Coordenação Geral da Política de Recursos Humanos. Políticas de recursos humano em saúde. [online] Brasília (DF), 2002. Disponível em: http:/ /www.opas.org.br/sistema/arquivos/politrh.Pdf > (19 set. 2003).

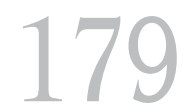

Rev Esc Enferm USP 2005; 39(2):173-80. 
Anaclara F. Veiga Tipple Thays Ribeiro de Souza Ana Lúcia Queiroz Bezerra Denize Boutellet Munari
(13) Sordi MRL, Bagnato MHS. Subsídios para uma formação profissional crítico reflexiva na área da saúde: o desafio da virada do século. Rev Lat-Am Enferm 1998; 6 (2):84-5.

(14) Brandt MJCG, Magalhães ZR. Composição da força de trabalho versus formação de pessoal em enfermagem no Brasil: reflexos na prática. Belo Horizonte: [s.n]; 1992.

(15) Antunes AV, Trevisan MA. Gerenciamento de qualidade: utilização no serviço de enfermagem. Rev Lat-Am Enferm 2002; 8 (2):35-44.

(16) Biasoto JG. Recursos humanos e qualificação profissional: impasses e possibilidades. São Paulo: Rev Formação 2002; 1(5):75-85.

(17) Machado MH. Gestão do trabalho em saúde no contexto de mudanças. São Paulo: Rev Adm Pública 2000; 34(4):133-46.

18) Brassard M. Qualidade: ferramentas para uma melhoria contínua. Rio de Janeiro: Qualitymark; 1994.

19) Neves AJ, Mota KM. Características específicas da implementação de estratégias de melhoria na gestão da saúde. Rev Adm Saúde 2002; 4(16): 33-8.

20) Associação Brasileira de Enfermagem.(ABEn) Caderno de Legislação/Documentos: a nova lei do exercício profissional da enfermagem. Brasília 1997; 1:1-36.

21) Conselho Federal de Enfermagem. Documentos básicos de enfermagem: principais leis e resoluções que regulamentam o exercício profissional de enfermeiros, técnicos e auxiliares de enfermagem. 2001. São Paulo: COREn-Seção SP. p. 142.
22) Prade SS. Controle de infecção hospitalar: uma revolução pela qualidade à assistência. [editorial] Rev Control Infec Hosp 1994; 1(1):3.

23) Tipple AFV, Pereira MS, Hayashida M, Moriya TM, Souza ACS. O ensino do controle de infecção: um ensaio teórico prático. Rev Lat-Am Enferm 2003; 11(2):245-50.

24) Cardo D. Controle de infecção requer empenho de hospitais e governos. Rev Meio Cult 2001; 4(16):8-10.

25) Ministério da Saúde. Qualificação profissional e saúde com qualidade. Rev Formação 2001; 1(1):5-7.

26) Bezerra ALQ. O contexto de educação continuada na enfermagem. São Paulo: Martinari; 2003.

27) Motta PR. Desempenho em equipes de saúde: manual. Rio de Janeiro: FGV; 2001.

28) Lima MAC, Cassiani SHB. Pensamento crítico: um enfoque na educação de enfermagem. Rev LatAm Enferm 2000; 8(1):23-30.

29) Galvão S. Identificação do modelo brasileiro de controle da infecção hospitalar. Âmbito Hosp 2002; 9:155

30) Galkin V. O papel das organizações governamentais e não-governamentais no controle de infecção hospitalar na Rússia. Rev Control Infec Hosp 1995; 2(2):44. [resumo apresentado na conferência $3{ }^{\text {rd }}$ International Society of Hospital Infection; 1994 sep 3-8; London].

31) Leite MJ, Pereira LL. Educação continuada em enfermagem. In: Kurcgant P., coordenadora. Administração em enfermagem. São Paulo: EPU; 1991. p. 147-63. 